\title{
An Analysis on Stability and Deposition Zones of Natural Gas Hydrate in Dongsha Region, North of South China Sea
}

\author{
Zuan Chen, ${ }^{1}$ Wuming Bai, ${ }^{1}$ Wenyue $X u,{ }^{2}$ and Zhihe Jin ${ }^{3}$ \\ ${ }^{1}$ Key Laboratory of the Study of Earth's Deep Interior, Institute of Geology and Geophysics, Chinese Academy of Sciences, \\ Beijing 100029, China \\ ${ }^{2}$ School of Earth and Atomospheric Sciences, Georgia Institute of Technology, Atlanta, GA 30332, USA \\ ${ }^{3}$ Department of Mechanical Engineering, University of Maine, Orono, ME 04469, USA \\ Correspondence should be addressed to Zuan Chen, zachen@mail.igcas.ac.cn
}

Received 18 March 2009; Revised 2 November 2009; Accepted 26 December 2009

Academic Editor: Costas Tsouris

Copyright ( $) 2010$ Zuan Chen et al. This is an open access article distributed under the Creative Commons Attribution License, which permits unrestricted use, distribution, and reproduction in any medium, provided the original work is properly cited.

We propose several physical/chemical causes to support the seismic results which find presence of Bottom Simulating Reflector (BSR) at site 1144 and site 1148 in Dongsha Region, North of South China Sea. At site 1144, according to geothermal gradient, the bottom of stability zone of conduction mode is in agreement with BSR. At site 1148, however, the stability zone of conduction mode is smaller than the natural gas presence zone predicted by the BSR. We propose three causes, that is, mixed convection and conduction thermal flow mode, multiple composition of natural gas and overpressure in deep sediment to explain the BSR presence or gas hydrate presence. Further, our numerical simulation results suggest yet another reason for the presence of BSR at site 1144 and site 1148. Because the temperatures in deep sediment calculated from the mixed convection and conduction thermal flow mode are lower than that from the single conduction mode, the bottom of gas hydrate stability zone (GHSZ) is deeper than the bottom of gas hydrate deposition zone (GHDZ) or BSR. The result indicates that occurrence zone of natural is decided by the condition that natural gas concentrate in the zone is greater than its solubility.

\section{Introduction}

Gas hydrate is an ice-like crystalline mineral in which hydrocarbon and nonhydrocarbon gases are held within rigid cages of water molecules [1]. Geological, geophysical, and geochemical evidence of gas hydrate is reported from 81 localities worldwide onshore in Arctic regions and offshore in passive and active margins and inland seas and lakes [1]. Several recent legs of the Ocean Drilling Program (ODP) have targeted known hydrate locations with the goal of characterizing marine gas hydrate [2]. The data collected from these studies provide a clearer picture of hydrate occurrence on both active and passive continental margins. On the basis of these data, attempts have been made to extrapolate local estimates of hydrate volume to infer a global inventory.

The South China Sea is the largest marginal sea in the western Pacific, and is well known for its abundant oil and gas reserves. A broad and wide continental slope $210,000 \mathrm{~km}^{2}$ in area extends to the northern parts of the South China Sea, and is a good site for gas hydrate formation and conservation.
Furthermore, a bottom simulating reflector (BSR) has been found on the Northern slope [3].

Site 1144 and Site 1148 which will be discussed in this paper are located in Dongsha region, north of South China Sea and at the lowermost continental slope off southern China (Figure 1). By using seismic, sonic logging, and geothermal data the distribution characteristics of gas hydrate in this zone were studied. BSR and amplitude blanking zone were discovered in seismic profiles. High-velocity interval and velocity reverse were distinguished in sonic logging curve at both sites. As indicated below, GHSZ based on conduction thermal flow model and BSR at site 1144 are basically in agreement, while GHSZ calculated from geothermal gradient using the conduction model is much smaller than the BSR predicated from seismic method at site 1148 .

In this paper, we aim to explain the results obtained by seismic methods. We first discuss several reasons to explain the difference at site 1148 from view of GHSZ. Second, we performe a numerical analysis to investigate the possible causes, based on a one-dimensional model of gas 


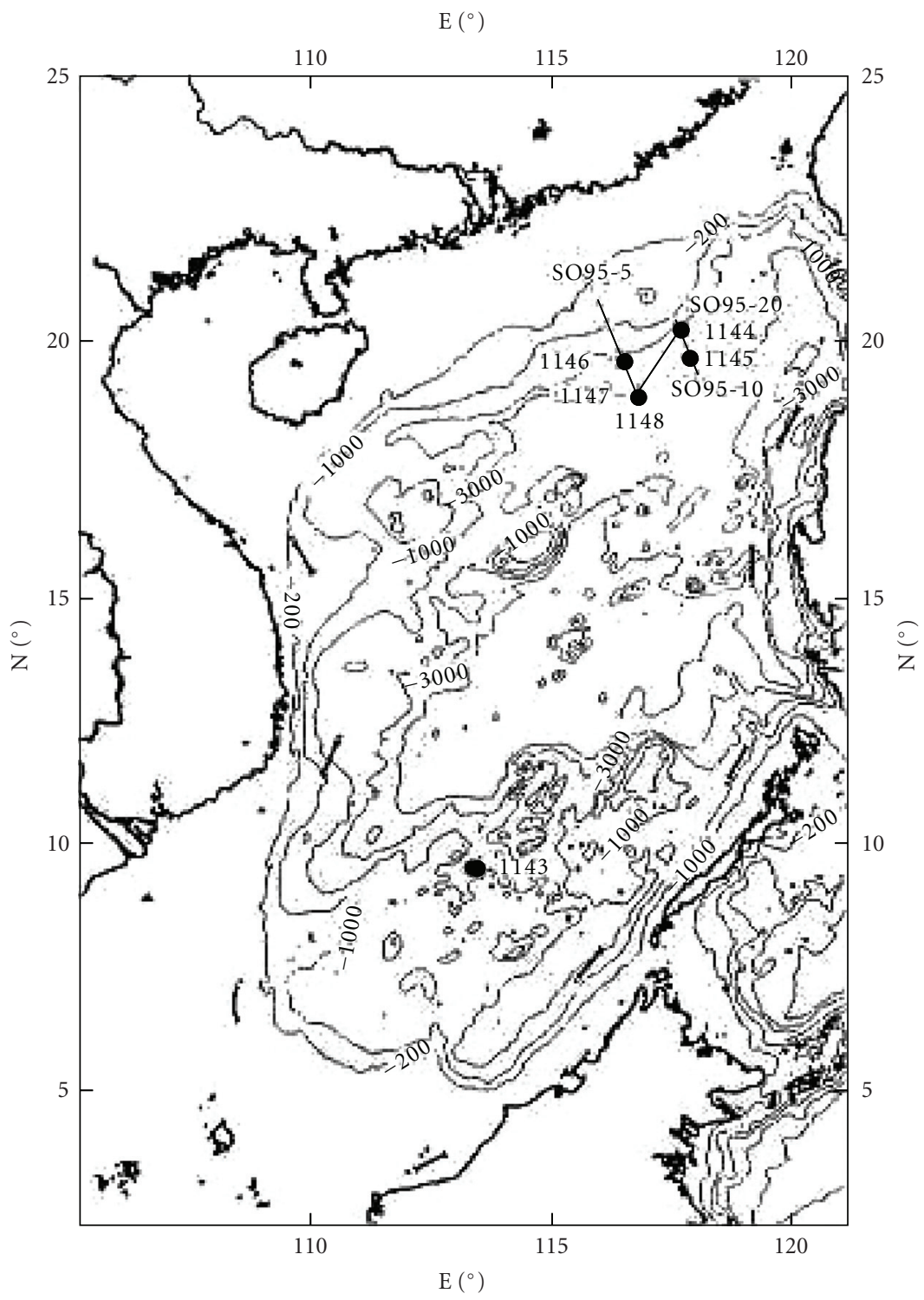

FIgURE 1: The sit 1148 in Dongsha region north of South China Sea.

hydrate formation and evolution in which effects of multiple composition in the gas are considered. Third, by comparing the seismic results and simulation analysis, we attempt to find available reason to strengthen our confidence on the presence of gas hydrate at site 1144 and site 1148 in Dongsha region north of South China Sea. In our calculations, based on the mixed convection and conduction thermal flow mode, GHSZ at both sites is found much deeper than GHDZ, which is ensured to be in agreement with BSR.

\section{Analysis about Bottom Boundary of GHSZ at Site 1144}

The water depth at site 1144 is $2037 \mathrm{~m}$. The bottom boundary of the gas (gas hydrate stability zone) GHSZ is predicted to be $720 \mathrm{~m}$ below sea floor at site 1144 based on the measured thermal gradient of $24^{\circ} \mathrm{C} / \mathrm{km}$ and a bottom-water tempera- ture of $3.1^{\circ} \mathrm{C}[3]$. Meanwhile, on the basis of data provided by the Ocean Drilling Program (ODP), the presence of gas hydrate in deep-sea sediments can be detected mainly based on the presence of a bottom-simulating reflector (BSR) on seismic profiles, which corresponds to the base of the gas hydrate deposition zone (GHDZ). The BSR depth at site 1144 has been found to be 730 meters below sea floor from seismic profiles, which is in agreement with the result obtained from thermal data [3].

\section{Analysis about Bottom Boundary of GHSZ at Site 1148}

The water depth at site 1148 is $3294 \mathrm{~m}$. The thermal gradient is $83^{\circ} \mathrm{C} / \mathrm{km}$ and the bottom-water temperature is $3.5^{\circ} \mathrm{C}$ [3]. Downhole and bottom-water temperature measurements at Site 1148 yielded a thermal gradient of $83^{\circ} \mathrm{C} / \mathrm{km}$, which is 
consistent with the location and water depth [3]. The bottom boundary of the gas (gas hydrate stability zone) GHSZ is predicted to be $250 \mathrm{~m}$ below sea floor at the site based on the temperature (thermal conduction mode), pressure (water depth), and phase equilibrium curve. The BSR depth at site 1148 has been found to be 475 meters below sea floor from seismic profiles, which is not in agreement with the result obtained from thermal data [3]. The possible reasons causing the difference are suggested as follows. (1) Thermal gradient is so big that it is not reliable, which may be excluded according to the reference from Song et al. [3]. (2) The multiple composition in the gas must be considered in the calculation of GHDZ. The temperature at the base of the gas hydrate stability zone may be a few degrees higher than that of methane with pure water. The presence of other gases can make the gas hydrate more stable. For example, ethane and propane contained in the gases have distinguished impact on the stability curve. (3) In a conductive model, thermal gradients remain constant for constant conductivity. Convection, by its nature, tends to increase temperature in the upper part of a system as temperatures in the lower part decrease [4]. It seems more reasonable to assume conduction-convection model rather than a conduction only model for calulating the steadystate temperature distribution. (4) The presence of a deep overpressure along the nearby northern shelf of South China Sea will increase the stability temperature, and a deeper base of GHSZ will be anticipated.

3.1. Thermal Gradient. According to the geothermal study, the thermal gradient is $83^{\circ} \mathrm{C} / \mathrm{km}$ and the water temperature at the sea floor is $3.5^{\circ} \mathrm{C}$ at site 1148 . The thermal gradient value is much larger than that in the neighbor area. The bottom boundary of the (gas hydrate stability zone) GHSZ is predicted to be $250 \mathrm{~m}$ at site 1148 from the geothermal data under hypothesis of pure water and single composition methane, which is much shallower than $475 \mathrm{~m}$ obtained from seismic method (Figure 2). It is possible to explain the difference between geothermal study and seismic result by thermal conductive model.

3.2. Thermal Flow Mode. The bottom boundary of the GHSZ is determined by comparing the geothermal curve and phase equilibrium curve. The geothermal curve is calculated based on the conduction thermal flow mode in which the temperature is dependent on thermal gradient as shown in Figure 2. Moreover, with a higher total heat flux, the enhanced fluid flow can result in a much lower temperature in deeper sediment while still being consistent with the shallow geothermal data. In the case, thermal flow mode is mixed conduction and convection mode, and the stability boundary will be moved to deeper sediment.

The problem of 1-D steady heat transport due to both fluid flow and heat conduction can be expressed mathematically [5] as follows:

$$
\begin{gathered}
\rho_{f} C_{f} V_{z} T-\lambda \frac{d T}{d z}=q_{e}, \\
T(0)=T_{0},
\end{gathered}
$$

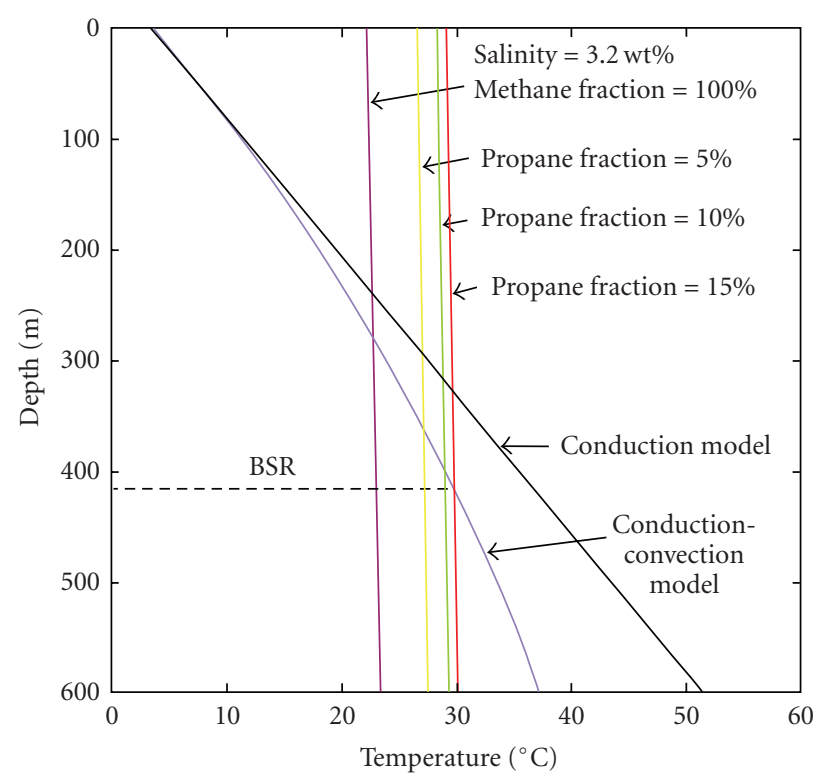

FIGURE 2: Stability zone predicated from different thermal flow modes and different fraction of methane and propane.

where $T$ is the temperature, $z$ is the vertical depth; $V_{z}$ is the vertical permeation velocity of ground fluid; $\rho_{f}, C_{f}$, and $\lambda$ represent fluid density, fluid specific heat and thermal conductivity, respectively; $T_{0}$ is the temperature on the top boundary or sea bottom; $q_{e}$ is constant total heat flux, which can in principle be determined by measuring the temperature, fluid flow velocity, and conductive heat flow at the surface $(z=0)$.

The solution of this problem is

$$
T(z)=T_{0} e^{\beta z}+\frac{q_{e}}{\lambda \beta}\left(1-e^{\beta z}\right),
$$

where

$$
\beta=\frac{\rho_{f} C_{f} V_{z}}{\lambda} .
$$

Figure 2 shows the calculated geothermal curves under a set of parameters including the vertical permeate velocities of ground fluid and bottom thermal flux. Meanwhile, the stability zone boundaries are obtained under the hypothesis of pure water and single composition methane. Apparently, the bottom boundary of the stability zone is lower than that obtained based on the single conduction mode, which may be the major reason for the discrepancy between the predication of the stability boundary and the anomalies in organic carbon gas contents and geochemical compositions. The gas hydrates containing ethane and propane in addition to methane may also be responsible for the discrepancy.

3.3. Multiple Compositions of Natural Gas. As we know, the origins of the gases in hydrate are both thermogenic and biogenic. The gas component from biogenic origin is almost all methane. The gas components from thermogenic origin are manifold. Besides methane, the possible components are 
ethane, propane, and so on. A lot of research works about thermogenic gas hydrates on the Gulf of Mexico continental slope have been carried out. The chronological framework of the upper 837.11-m composite section ( 2 32.7 Ma to present) at sit 1148 in Dongsha region north of South China Sea has been set up based on biostratiagraphy and magnetostratigraphy [6]. According to the long-term observations of anomalously increased sea-surface temperature scanned by satellite-based thermal infrared and the investigations of the gas geochemistry of bottom water, the authigenic minerals, and the fluid composition, it was concluded that there exist strong degassing and hydrocarbon fluid activities in the submarine. The main composition of submarine gas in the northern continental slopes of the South China Sea is $\mathrm{CH}_{4}$ and thermogenic [7].

The propane composition has the larger influence on equilibrium temperature comparing with other gas compositions except methane [8]. Hence, two compositions, that is, methane and propane, are considered in our model. Equilibrium temperature of gas hydrate system with both methane and propane increases with pressure and propane fraction, and decreases with salinity of the coexisting liquid solution. The equilibrium temperature is assumed as to be a function of pressure, salinity, and fraction of propane, and is regressed from data obtained using the software CSMHYD [8] as follows:

$$
\begin{aligned}
T= & \frac{1000}{K}-273.15 \\
K= & a_{0}(h, s)+a_{1}(h, s) \log (p)+a_{2}(h, s) \log (p)^{2} \\
& +a_{3}(h, s) \log (p)^{3}+a_{4}(h, s) \log (p)^{4}, \\
a_{1}(h, s)= & b_{i 0}(s)+b_{i 1}(s) h+b_{i 2}(s) h^{2} \\
& +b_{i 3}(s) h^{3}+b_{i 4}(s) h^{4}, \quad(i=0,1,2,3,4),
\end{aligned}
$$

where $T$ is temperature, $p$ is pressure, $h$ is fraction of propane in natural gas, and $s$ is salinity.

Figure 2 indicates that phase equilibrium curves change with the fraction of propane. Apparently, the stability boundary will be moved to deeper sediment, and the change is obvious. When propane fraction reaches $10 \%$, the depth of bottom stability boundary is $475 \mathrm{~m}$, which agrees with the BSR result. Moreover, the hypothesis that propane and other gas composition exist in natural gas in addition to methane in site 1148 is strongly supported by the measurements of organic carbon gases, which may indicate hydrate forming gases carried by the fluid flow from a deep source [9].

3.4. Overpressure under Deep Sediment Region. Deposit environment in the north aktian zone of South China Sea is mainly shore, infraneritic, and bathyal region. The emptied terrigenous material is rich, and the laid down velocity is high. The condition is very prominent after Miocene epoch. Therefore, a deep overpressure by rapid sedimentation along the nearby northern shelf of the South China Sea can be another cause for enhancing phase equilibrium temperature and a high upward fluid flow. Of course, we do not have direct insite measured data to support the hypothesis. If the convection thermal flow mode at site 1148 is possible, it will be indirect evidence to indicate overpressure existence in the region. Moreover, the stability temperature only increases $3.5^{\circ} \mathrm{C}$ when the pressure increases $10 \mathrm{MPa}$. That means overpressure in deep sediment is not a major factor affecting the boundary of hydrate deposition.

\section{Numerical Simulation of GHSZ and GHDZ at Site 1144 and Site 1148}

To carry out numerical simulation for the dynamic process of hydrate in marine sediment, we need (1) to create phaseinversion model of natural gas hydrate, which concerns the pressure, temperature, and salinity of the fluid, as well as phase equilibrium condition, solubility, fluid density, and enthalpy. The simulation results would be unreliable if the dynamic process of hydrate phase-inversion was not considered precisely, and (2) to create a general mathematical model which can describe the transport process of fluid, heat, natural gas, and salt, as well as some geological processes, such as sedimentation and natural gas formation resource.

Stability zone and occurrence zone of methane hydrate formation can be predicted under certain boundary conditions using the calculation model of methane hydrate in seafloor sediment presented by Xu et al. [10-12]. Stability zone is decided by intersection of phase transition curve and actual ground temperature curve. Gas hydrate occurrence zone must lie in stability zone, and is decided by the condition that gas concentration is greater than its solubility. So prediction of stability zone and occurrence zone of gas hydrate formation will be effected by the distribution of actual temperature and pressure as well as mass flow and gas flow at the boundary.

The phase transition process, that is, the phase equilibrium relationship of hydrate formation process, was considered by Xu et al. [11, 12], and Chen et al. [13], and the formation or dissolution was described dynamically through enthalpy change. The calculation method of phase transition in this paper is the same as that used in Xu et al. $[11,12]$ and Chen et al. [13]. In fact, natural gas with certain proportion of compositions usually changes their proportions in hydrate after phase transition, that is, composition proportion of natural gas will also change. We do not take into account the complex process and assume simply that composition proportion of natural gas is fixed.

4.1. Model Formulation. The mathematical model formulation used in the calculation is summarized as follows [11$13]$.

Assuming natural gas diffusion occurs only in the liquid phase, the transport of natural gas can be described by

$$
\frac{\partial(\phi \rho C)}{\partial t}+\frac{\partial}{\partial z}\left(q_{l} C_{l}+q_{g} C_{g}+q_{h} C_{h}-\phi S_{l} \rho_{l} D_{c} \frac{\partial}{\partial z} C_{l}\right)=Q
$$

where $t$ is the time, $D_{C}$ is the diffusivity of natural gas in the liquid solution, $Q$ represents the rate of in situ natural 
TABLe 1

\begin{tabular}{cl}
\hline Parameter & Value \\
\hline $\mathrm{g}$ & $9.81 \mathrm{~ms}^{-2}$ \\
$\phi$ & 0.5 \\
$k$ & $1 \times 10^{-16} \mathrm{~m}^{2}$ \\
$\rho_{h}$ & $920.0 \mathrm{kgm}^{-3}$ \\
$\lambda$ & $1.0 \mathrm{Wm}^{-1} \mathrm{C}^{-1}$ \\
$C_{s}$ & $1000.0 \mathrm{Jkg}^{-1} \mathrm{C}^{-1}$ \\
$\mu_{l}$ & $0.888 \times 10^{-3} \mathrm{kgm}^{-1} \mathrm{~s}^{-1}$ \\
$D_{c}$ & $1.0 \times 10^{-9} \mathrm{~m}^{2} \mathrm{~s}^{-1}$ \\
\hline
\end{tabular}

gas production, subscripts $l, g$, and $h$ represent liquid, gas, and hydrate, respectively, $\phi$ is porosity; $\rho, C$, and $q$ are the density, natural gas concentration, and Darcian flow rate, respectively; $S$ is the saturation, and $z$ is the spatial coordinate pointing upward.

Fluid flow is assumed to obey Darcy's law, that is,

$$
\begin{aligned}
q_{l} & =-\frac{k k_{l} \rho_{l}}{\mu_{l}}\left(\frac{\partial}{\partial z} p+\rho_{l} g\right), \\
q_{g} & =-\frac{k k_{g} \rho_{g}}{\mu_{g}}\left(\frac{\partial}{\partial z} p+\rho_{g} g\right),
\end{aligned}
$$

where $g$ is the gravitational acceleration, $k$ is the permeability of the porous medium, $P$ is the pressure, $\mu$ is the fluid viscosity, and $k_{l}$ and $k_{g}$ are relative permeability of liquid and gas.

Conservation of energy is cast in terms of enthalpy $H$ and temperature $T$ and written as

$$
\begin{aligned}
& \frac{\partial}{\partial t}\left[\phi \rho H+(1-\phi) \rho_{s} H_{s}\right]+\frac{\partial}{\partial z}\left[q_{l} H_{l}+q_{g} H_{g}+q_{h} H_{h}-\lambda \frac{\partial}{\partial z} T\right], \\
& \quad=0,
\end{aligned}
$$

where subscript $s$ refers to the sediment matrix, $C_{s}$ denotes specific heat capacity, and $\lambda$ is effective thermal conductivity.

4.2. Numerical Calculation of GHSZ and GHDZ around Site 1144. Seafloor sediment with $800 \mathrm{~m}$ thickness is accounted, and 401 junction points are marked. Table 1 lists the physical parameters of the medium. The boundary conditions are as follows. Pressure in seafloor is $20.37 \mathrm{MPa}$, temperature in seafloor is $3.1^{\circ} \mathrm{C}$, weight percentage of natural gas content in seafloor is $10^{-6} \%$, weight percentage of salt in seafloor is $3.2 \%$, heat flow in the bottom of computed zone is $0.03 \mathrm{~W} / \mathrm{m}^{2}$, mass flow in bottom is $2 \times 10^{-7} \mathrm{~kg} / \mathrm{m}^{2} / \mathrm{s}$, natural gas flow in bottom is $3.035 \times 10^{-10} \mathrm{~kg} / \mathrm{m}^{2} / \mathrm{s}$, and salt flow in bottom $3.2 \times 10^{-9} \mathrm{~kg} / \mathrm{m}^{2} / \mathrm{s}$. The natural gas is pure methane. The above parameter values have eventually been determined through trials. The calculated GHSZ and GHDZ are shown in Figure 3, in which $z$ is depth from seafloor; bottom abscissa means temperature $T$, top abscissa is natural gas content $C$. The results indicate that when the mass flow in bottom is large enough, the GHDZ will exist in sediment and its bottom depth $720 \mathrm{~m}$ will be consistent with BSR

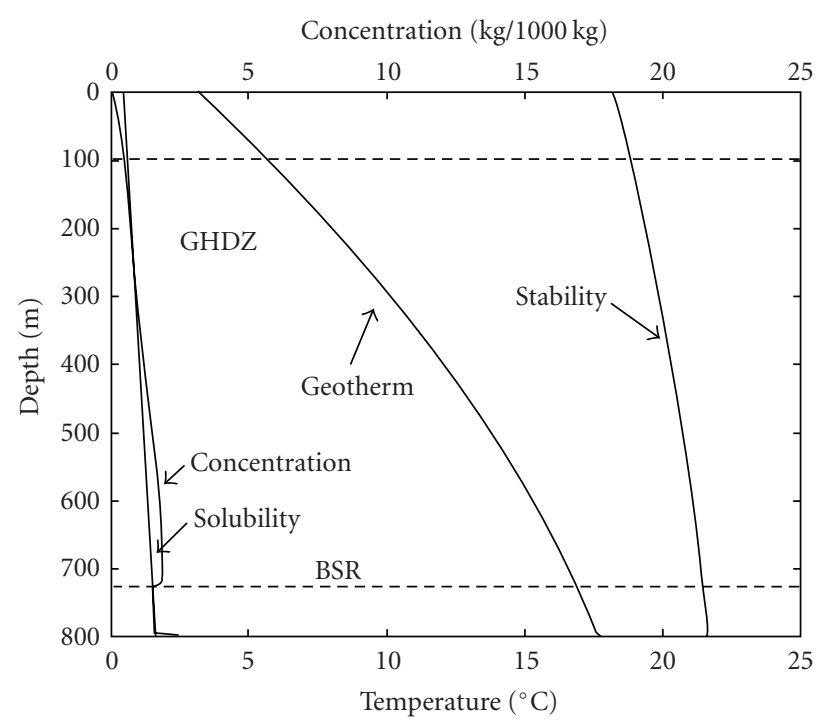

FIGURE 3: Numerical simulation for GHSZ and GHDZ at site 1144.

from seismic method. In this case, a mixed conduction and convection thermal flow mode is used, and the stability boundary will be moved to deeper sediment than the bottom boundary of GHDZ.

4.3. Numerical Calculation of GHSZ and GHDZ around Site 1148. Seafloor sediment with $600 \mathrm{~m}$ thickness is accounted, and 301 junction points are marked. Boundary conditions are pressure in seafloor of $32.94 \mathrm{MPa}$, temperature in seafloor is $3.5^{\circ} \mathrm{C}$, weight percentage of natural gas content in seafloor is $10^{-6} \%$, weight percentage of salt in seafloor is $3.2 \%$, heat flow in bottom of computed zone is $0.06 \mathrm{~W} / \mathrm{m}^{2}$, mass flow in bottom is $1.5 \times 10^{-7} \mathrm{~kg} / \mathrm{m}^{2} / \mathrm{s}$, natural gas flow in bottom is $3.035 \times 10^{-10} \mathrm{~kg} / \mathrm{m}^{2} / \mathrm{s}$, and salt flow in bottom is $3.2 \times 10^{-9} \mathrm{~kg} / \mathrm{m}^{2} / \mathrm{s}$. The natural gas is composed of methane $(88 \%)$ and propane $(12 \%)$. Through many times trial, the parameter values mentioned above have eventually been determined. The calculated GHSZ and GHDZ are shown in Figure 4 . The results indicate that when the mass flow in bottom is large enough, the GHDZ will exist in sediment and its bottom depth $475 \mathrm{~m}$ will be consistent with BSR from seismic method. Again, a mixed conduction and convection thermal flow mode is used, and the stability boundary will be moved to deeper sediment than the bottom boundary of GHDZ just as site 1144 .

\section{Conclusion}

In this paper, we propose several possible reasons to support the seismic method results which find presence of BSR at site 1144 and site 1148. At site 1144, according to geothermal gradient, the bottom of stability zone of conduction mode is in agreement with BSR or $720 \mathrm{~m}$ depth bellow seafloor. At site 1148, due to the high geothermal gradient, the stability zone of conduction mode is less than the natural gas presence zone predicted by BSR which is $475 \mathrm{~m}$ below seafloor. BSR presence or gas hydrate presence may be explained by the 


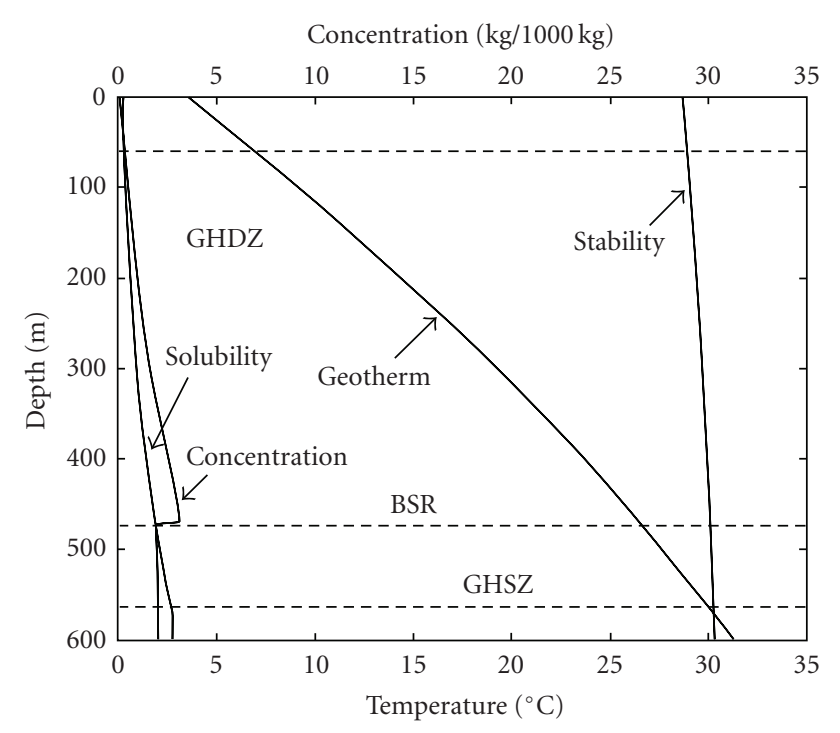

FIGURE 4: Numerical simulation for GHSZ and GHDZ at site 1148.

following three mechanisms, that is, mixed conduction and convection thermal flow mode, multiple composition in natural gas, and overpressure in deep sediment. Logging result in the region [3] (Song, 2001) also indicate that bottom boundary of GHSZ at site 1148 is at $475 \mathrm{~m}$. We numerically simulate the dynamic process of gas hydrate evolution at site 1144 and site 1148, and GHDZ will reach $720 \mathrm{~m}$ and $475 \mathrm{~m}$, respectively. Moreover, numerical results also indicate that in the two cases, thermal flow is mixed conduction and convection mode, and GHSZ is deeper than GHDZ. These results also indicate that GHSZ is a basic condition for presence of GHDZ, which is finally predicted by the mathematical model considering the dynamic process of hydrate in marine sediment.

It should be pointed out that our conclusions are only theoretical inference in order to explain the seismic results. Real situations at site 1144 and 1148 need more direct investigation.

\section{Acknowledgment}

The authors would like to thank two anonymous reviewers and the associated editor for their critical but helpful comments. The research work is supported by CNSF (40274026, 40874046).

\section{References}

[1] E. D. Sloan Jr., "Fundamental principles and applications of natural gas hydrates," Nature, vol. 426, no. 6964, pp. 353-359, 2003.

[2] C. K. Paull, R. Matsumoto, and P. Wallace, "Leg 164 science party," in Proceedings of the Ocean Drilling Program, vol. 164 of Initial Reports, College Station, Tex, USA, 1996.

[3] H.-B. Song, J.-H. Geng, H.-K. Wang, et al., "A preliminary study of gas hydrates in Dongsha Region North of South China Sea," Chinese Journal of Geophysics, vol. 44, no. 5, pp. 687-795, 2001 (Chinese).
[4] R. W. Rex and D. J. Howell, "Assessment of U.S. geothermal resources," in Geothermal Energy, P. Kruger and C. Otte, Eds., 1973.

[5] J. D. Bredehoeft and I. S. Papadopulos, "Rates of vertical groundwater movement estimated from the earth's thermal profile," Water Resources Research, vol. 1, no. 2, pp. 325-328, 1965.

[6] P. Wang, W. L. Prell, P. Blum, et al., "Leg 184," in Proceedings of the Ocean Drilling Program, vol. 184 of Initial Reports, College Station, Tex, USA, 2000.

[7] B. Wu, G. Zhang, Y. Zhu, et al., "Progress of gas hydrate investigation in China offshore," Earth Sciences Frontiers, vol. 10, no. 1, pp. 177-189, 2003 (Chinese).

[8] E. D. Sloan Jr., Clathrate Hydrates of Natural Gases, Marcel Dekker, New York, NY, USA, 1998.

[9] Y. Zhu, Y. Huang, M. Ryo, and B. Wu, "Geochemical and stable isotopic composition of pore fluids and authigenic siderite concretions from site 1146,ODP leg 184: implications for gas hydrate," in Proceedings of the Ocean Drilling Program, Scientific Results, W. L. Prell, P. Wang, P. Blum, D. K. Rea, and S. C. Clemens, Eds., vol. 184 of Initial Reports, pp. 1-15, 2000.

[10] W. Xu and C. Ruppel, "Predicting the occurrence, distribution, and evolution of methane gas hydrate in porous marine sediments," Journal of Geophysical Research B, vol. 104, no. 3, pp. 5081-5095, 1999.

[11] W. Xu, R. P. Lowell, and E. T. Peltzer, "Effect of seafloor temperature and pressure variations on methane flux from a gas hydrate layer: comparison between current and late Paleocene climate conditions," Journal of Geophysical Research $B$, vol. 106, no. 11, pp. 26413-26423, 2001.

[12] W. Xu, "Modeling dynamic marine gas hydrate systems," American Mineralogist, vol. 89, no. 8-9, pp. 1271-1279, 2004.

[13] Z. Chen, W. Bai, and W. Xu, "Prediction of stability zones and occurrence zones of multiple composition natural gas hydrate in marine sediment," Chinese Journal of Geophysics, vol. 48, no. 4, pp. 936-945, 2005 (Chinese). 

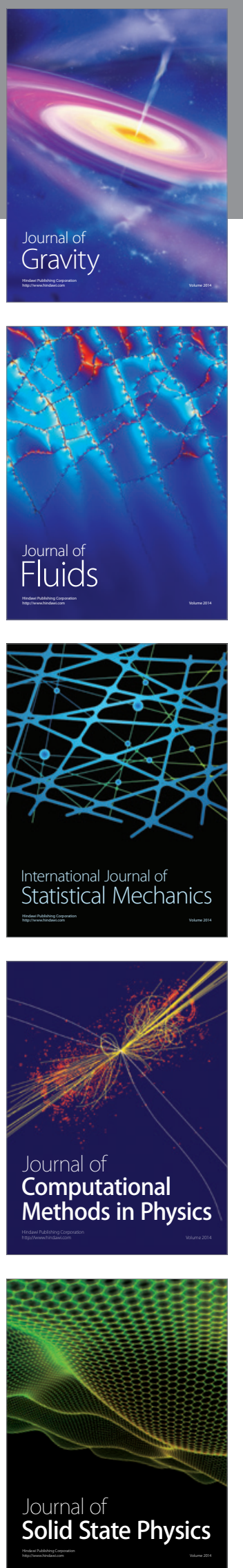

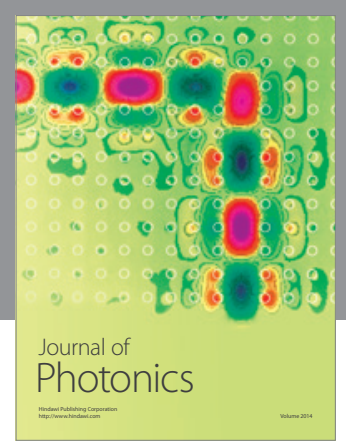

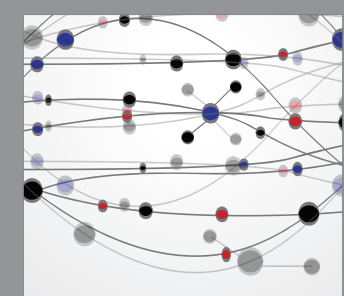

The Scientific World Journal
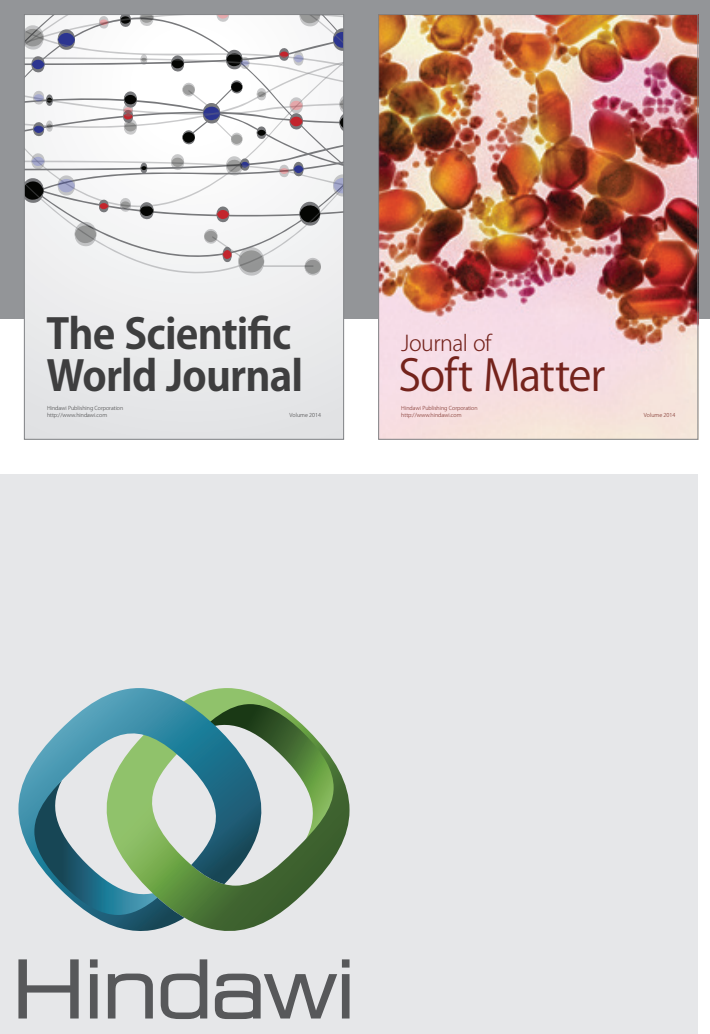

Submit your manuscripts at

http://www.hindawi.com
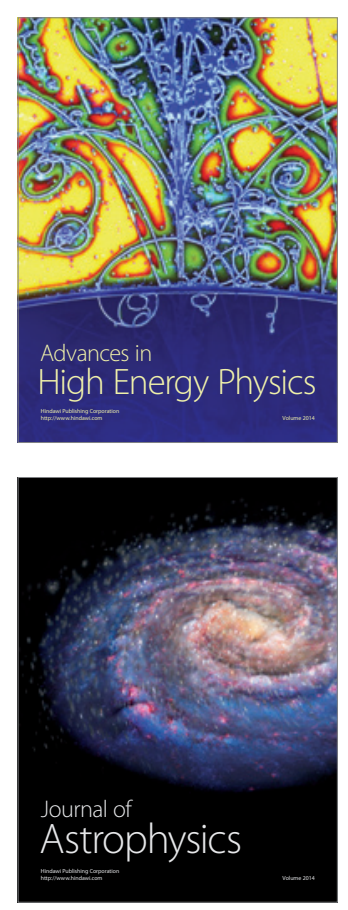
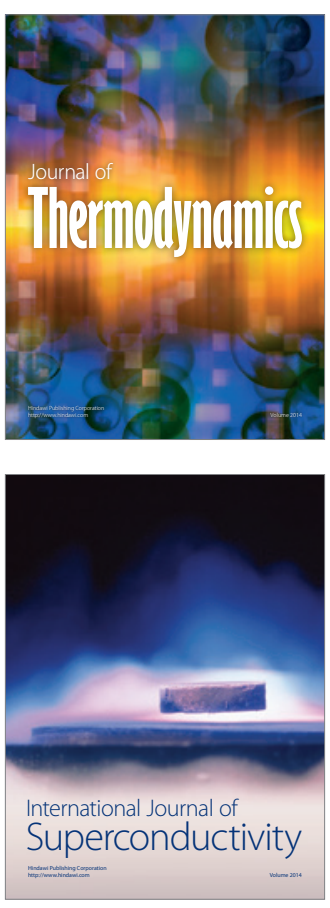
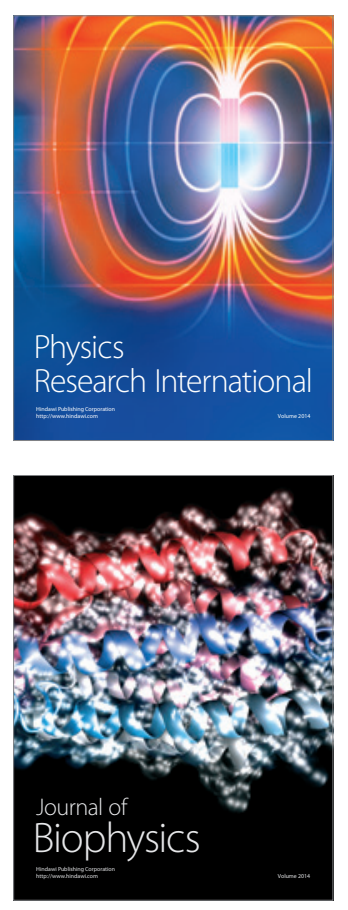
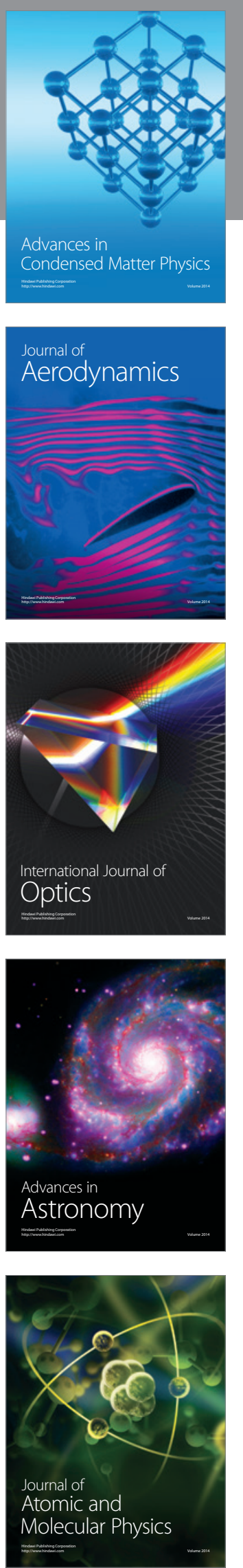